\title{
La Réserve naturelle nationale du Platier d'Oye : analyse de vingt ans de conflits (1987-2007)
}

\author{
Michel Delsaut ${ }^{1}$, Catherine Meur-Férec $^{2}$ \\ 1 Biologiste, Université des sciences et technologies de Lille, Département Neurosciences, 59655 Villeneuve d'Ascq cedex, France \\ 2 Géographe, Université de Bretagne Occidentale, Équipe Géomer, UMR6554, LETG, IUEM, 29280 Plouzané, France
}

\author{
Mots-clés : \\ réserve naturelle; \\ chasse ; \\ érosion côtière ; \\ conflits ; \\ Nord-Pas-de-Calais
}

\section{Keywords:}

national natural reserve;

hunting;

coastal erosion;

governance;

win-win principle

\begin{abstract}
Résumé - Créée il y a 20 ans, la Réserve naturelle nationale du Platier d'Oye présente une remarquable diversité d'habitats littoraux et constitue un espace de nature résiduel sur la côte fortement industrialisée et urbanisée du Nord-Pas-de-Calais. Une grande diversité d'acteurs aux intérêts divergents entraîne de nombreuses concurrences d'usage et des conflits qui tendent à occulter les objectifs naturalistes de la réserve. L'absence d'un véritable arbitrage des priorités fait émerger deux problèmes majeurs : 1) la gestion de l'érosion côtière, pour laquelle les objectifs de la réserve et les intérêts des riverains suivent deux logiques antagonistes ; 2) la chasse illégale, perçue comme un droit d'usage en raison d'une tolérance de longue durée. Sur ce territoire fortement investi, un système de gouvernance favorisant le principe $\mathrm{du}$ « gagnant-gagnant » peut donner l'illusion qu'il est possible de résoudre les conflits sans assumer de véritables choix durables et raisonnés prenant en compte la «valeur nature » de ce patrimoine commun.
\end{abstract}

\begin{abstract}
The National Nature Reserve of Platier d'Oye: Analysis of twenty years of conflict (19872007). The National Nature Reserve of Platier d'Oye was established 20 years ago. It offers an outstanding diversity of littoral habitats and is a residual natural site on the heavily industrialized and urbanized coast of Nord-Pas-de-Calais (northern France). The wide variety of stakeholders with divergent interests generates numerous situations of competition and conflict which tend to overshadow the naturalist objectives of the reserve. The absence of any real arbitration to establish priorities brings to the foreground two major problem areas: 1) the management of coastal erosion, for which the objectives of the reserve and the interests of the local residents follow two opposing sets of logics and, 2) illegal hunting, perceived as a customary right due to long-standing acceptance of this practice. Within this heavily invested territory a system of governance based on the "win-win" principle may promote the illusion that conflicts can be resolved without adopting genuinely sustainable choices considering the "Nature value" of this shared heritage.
\end{abstract}

\section{Introduction}

Les problèmes de protection de l'environnement et de préservation de la diversité biologique sont de plus en plus intégrés à la réflexion politique. Parmi les moyens susceptibles de contribuer à ces objectifs, la mise en réserve de certains espaces «naturels » apparaît comme une procédure à la fois simple et efficace. En théorie, la protection par la loi et la mise en place de règles précises doivent effectivement garantir la sauvegarde des milieux et des espèces. Mais l'exemple de la Réserve naturelle

\footnotetext{
Auteur correspondant :

C. Meur-Férec, meurferec@univ-brest.fr
}

nationale du Platier d'Oye ${ }^{1}$ (créée en 1987), espace de nature résiduel sur une côte fortement industrialisée et urbanisée et qui, de ce fait, concentre des acteurs, des enjeux, des intérêts, des usages et des valeurs très différents, offre une belle opportunité d'analyse de la complexité que peut atteindre la gestion de tels territoires.

En effet, quel est le poids réel des différents acteurs, des différents intérêts ? Quel est l'effet des choix, ou des non-choix, en termes de gestion de cet espace? Quel mode de gouvernance (Gaudin, 2002 ; Theys, 2002) fonctionne sur ce territoire? Les conflits peuvent-ils être, localement, un mode de régulation sociale (Reynaud, 1999), ajustant le rôle de la puissance publique en matière d'organisation

${ }^{1}$ Les auteurs sont membres de son comité de gestion. 
des règles d'usage sur les biens collectifs environnementaux (Jeanneaux, 2006)? Enfin, cette régulation permetelle de respecter les objectifs de préservation et d'intérêt général affichés par la loi?

L'analyse de ce système complexe est fondée sur une approche de terrain et sur des données qui proviennent de sources variées : réunions du comité consultatif de gestion de la réserve, réunions de travail "spécialisées » organisées par les services de l'État (DIREN, préfecture...), comptes rendus et courriers suscités par ces rencontres, rapports élaborés depuis 20 ans, mémoires d'étudiants, jugements des tribunaux.

Entre Calais et Dunkerque, la Réserve naturelle nationale du Platier d'Oye (391 hectares, dont 250 hectares sur le domaine public maritime) borde la mer du Nord. Durant les années 1970, accompagnant la croissance économique (complexe industrialo-portuaire de Dunkerque, centrale nucléaire de Gravelines) et démographique, un lotissement d'environ 150 maisons a été construit au cœur des dunes du Platier d'Oye.

Plusieurs niveaux de protection se superposent sur le site. La partie terrestre cadastrée appartient au Conservatoire du littoral et des rivages lacustres et le statut de Réserve naturelle nationale s'applique aussi au-delà, sur la plage et sur une partie de l'estuaire de l'Aa. La réserve est également classée dans le réseau Natura 2000 comme zone de protection spéciale (FR.3110039) par la Communauté européenne au titre de la directive Oiseaux; elle se trouve en outre dans le périmètre de la zone nationale d'intérêt écologique faunistique et floristique (0073000).

L'intérêt écologique de la Réserve naturelle nationale du Platier d'Oye est qu'elle réunit une diversité remarquable d'habitats, répartis au sein de milieux complémentaires et fonctionnellement unis : l'estran sableux, l'estuaire, les dunes et les prairies humides (Fig. 1). Plusieurs de ces habitats sont classés dans l'annexe 1 de la directive européenne Habitats. Cette valeur est encore majorée par la situation particulière du Platier, véritable coupure verte entre deux pôles industriels et urbains importants (Dunkerque et Calais), point d'accueil et de nourrissage des oiseaux sur une voie essentielle de migration. La richesse avifaunistique du Platier d'Oye n'a d'ailleurs pas échappé aux chasseurs de gibier d'eau, qui, en s'installant à cet endroit, en ont reconnu la valeur exceptionnelle.

\section{Une réserve dans un contexte très particulier}

La gestion naturaliste appliquée depuis 20 ans (creusement de mares, fauche ou pâturage extensif, etc.) a renforcé les capacités d'accueil de la faune et de la flore. De nombreuses espèces migratrices : anatidés et limicoles, utilisent le relais ainsi offert et les espèces nicheuses sont nombreuses et parfois remarquables, comme la sterne caugek, la mouette mélanocéphale ou l'avocette élégante. Certaines sont très bien représentées (vanneau), d'autres, exceptionnelles (échasse blanche). Au total, près de 250 espèces d'oiseaux fréquentent le site, démontrant un effet positif des mesures de gestion. Il en est de même pour la flore, qui reflète la diversité des milieux rencontrés. On trouve ainsi des associations végétales caractéristiques à la fois : 1) des prairies arrière-littorales, avec leurs pelouses, une petite roselière ; 2 ) des dunes, avec leurs différentes facettes depuis la dune blanche jusqu'aux zones entièrement envahies par les argousiers ; 3) de l'estran ; 4) de l'estuaire, avec son cortège de plantes telle la rare obione pédonculée (Halimione pedunculata), dont environ cinq millions de pieds ont été notés dans la partie estuarienne du site.

Ces éléments positifs sont cependant contrebalancés par plusieurs facteurs résultant d'une histoire complexe. Le Platier d'Oye présente en effet un contexte socialement très sensible : un lotissement situé au cœur d'une réserve naturelle et menacé par l'érosion marine ; un espace littoral de loisirs et de tourisme dont l'accès se fait en traversant la réserve (et les dunes, qui constituent le dernier rempart contre la mer) ; et surtout une zone côtière d'intérêt écologique considérable, mais où la pratique de la chasse n'a pas cessé après le classement en réserve. Cet espace concentre donc concurrences d'usage, logiques antagonistes, échelles de réflexion spatiotemporelles divergentes qui, au gré des aménagements et des choix de gestion, ont abouti à des conflits violents et stérilisants. Cette situation a considérablement complexifié, au quotidien, la gestion de la réserve et, parfois, oblitéré ses principaux objectifs.

\section{Une grande diversité d'acteurs aux intérêts divergents}

La matrice présentée dans l'Encadré recense les différents intervenants et leurs interactions. Nous avons considéré le préfet et le sous-préfet comme des acteurs «à part », leur rôle, outre de faire respecter la loi, étant avant tout d'orchestrer les relations entre les acteurs, de les réunir et de limiter au maximum les conflits afin de préserver la paix sociale. Par ailleurs, faune et flore sont pris en compte en tant qu' « acteurs biologiques », car ce sont des êtres vivants qui justifient le classement de ce milieu en réserve et qui éventuellement le modifient.

\section{Une absence d'arbitrage des priorités}

Ces différents acteurs ont des logiques et des intérêts parfois divergents, qui interfèrent avec les objectifs de la réserve. Deux exemples sont particulièrement représentatifs. 


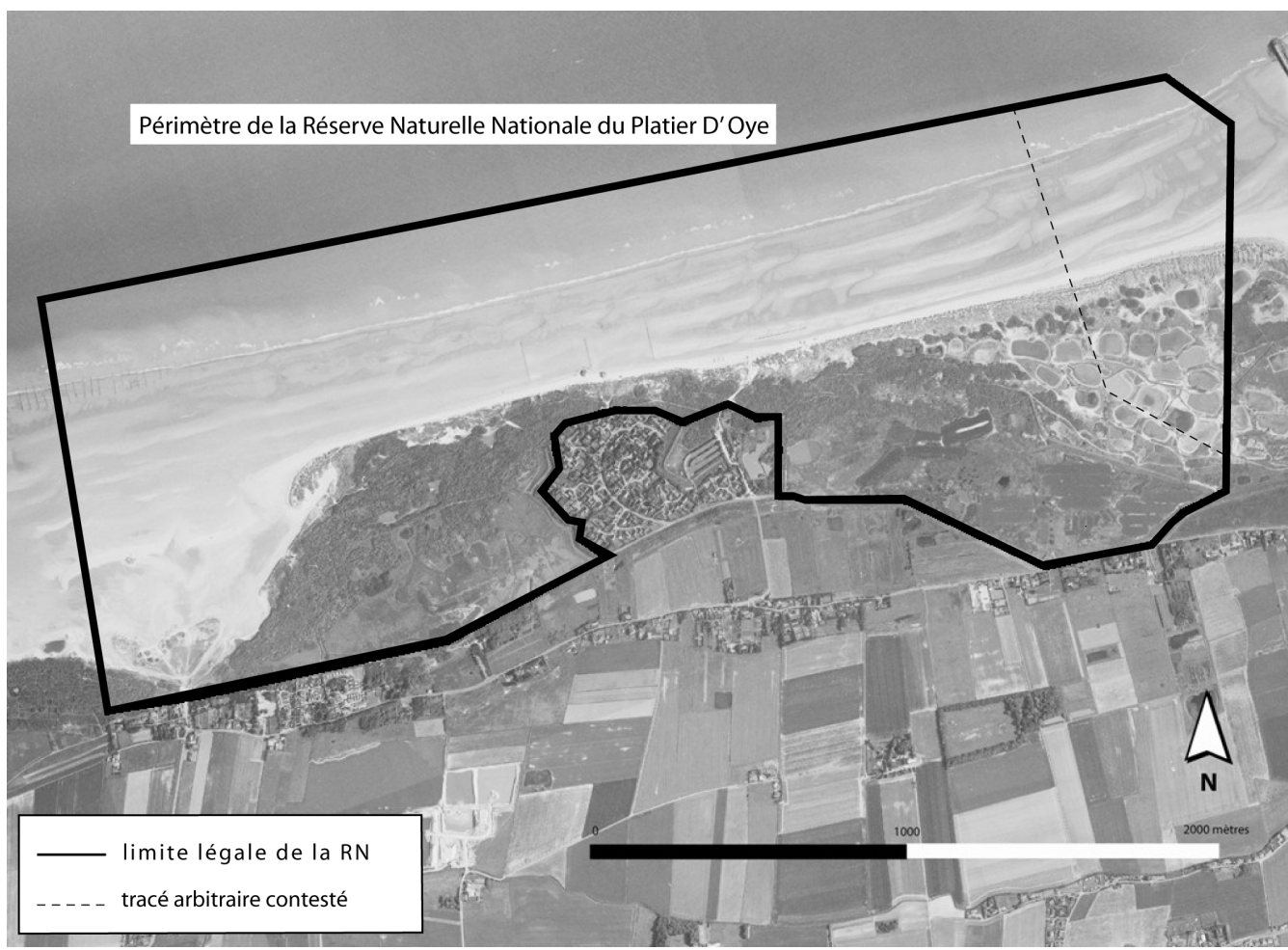

Fig. 1. Plan du Platier d'Oye. (Source : Plan de gestion du Platier d'Oye.)

\section{La protection du lotissement contre la mer}

L'érosion de l'étroit cordon dunaire, qui sépare de l'estran le secteur bâti situé en contrebas, risque d'entraîner d'importantes inondations marines. Face à cette situation, diverses méthodes expérimentales d'engraissement ont été testées (peignes hydrauliques, brise-vent et épis de bois). Mais la faiblesse du stock sableux et l'élévation prévue du niveau marin (IPCC, 2007) rendent, à long terme, ces solutions peu durables dans un secteur si vulnérable (Meur-Férec et al., 2008). En outre, ces travaux ont été réalisés sans une réelle prise en compte du statut de réserve (pas de mesure des impacts biologiques); c'est donc le statut de domanialité publique (DPM) qui a clairement primé, sans que la question de l'arbitrage des priorités n'ait même été soulevée.

En fait, deux logiques fondamentalement antagonistes se confrontent ici. D'une part, assurer la pérennité du lotissement constitue une priorité indiscutable pour les élus locaux ; d'autre part, combattre des phénomènes naturels, comme la mobilité du trait de côte, peut apparaître en contradiction avec les objectifs de la réserve. En effet, la mobilité du cordon dunaire, voire sa rupture, pourrait favoriser les intrusions d'eau marine dans la réserve et accroître son potentiel biologique. Sur d'autres sites de nature, la dépoldérisation volontaire par destruction de digues a effectivement été choisie pour ces mêmes raisons écologiques (Goeldner-Gianella, 2007).

\section{La question de la chasse dans la réserve}

L'histoire de la réserve est mouvementée dès l'origine. Le décret de création signé en 1987 (avec un an de retard pour cause de période électorale) est accompagné d'un plan dont il n'est pas fait mention dans le texte et que la plupart des intervenants concernés ne découvrent qu'après coup. Sur ce plan, une ligne brisée tracée au crayon déplace la limite orientale de la réserve pour exclure une grande partie de l'estuaire de l'Aa où sont situées de nombreuses huttes de chasse. Ce trait, probablement tracé dans un souci d'apaisement, puisqu'il permet la création de la réserve en préservant partiellement les intérêts des chasseurs, semble être le résultat d'une négociation menée dans un secret au moins partiel. Il va pourtant être à l'origine de bien des conflits entre chasseurs, usagers, associations de protection de la nature et gestionnaires.

Ainsi, suite au fameux trait de crayon, douze huttes normalement intégrées à la réserve en sont, de fait, exclues. Les autres, clairement situées dans la version amputée de la réserve, sont déplacées, au frais de l'État, mais rapidement détruites par une tempête; les chasseurs réinvestissent alors sept de leurs anciennes installations. Cette réoccupation est tolérée par les autorités dans un souci d'apaisement (décret municipal autorisant la chasse dans la réserve en 1990). Mais de nombreuses actions sont entreprises par les associations de protection de la 
Encadré. Matrice des acteurs et principaux types d'interrelations

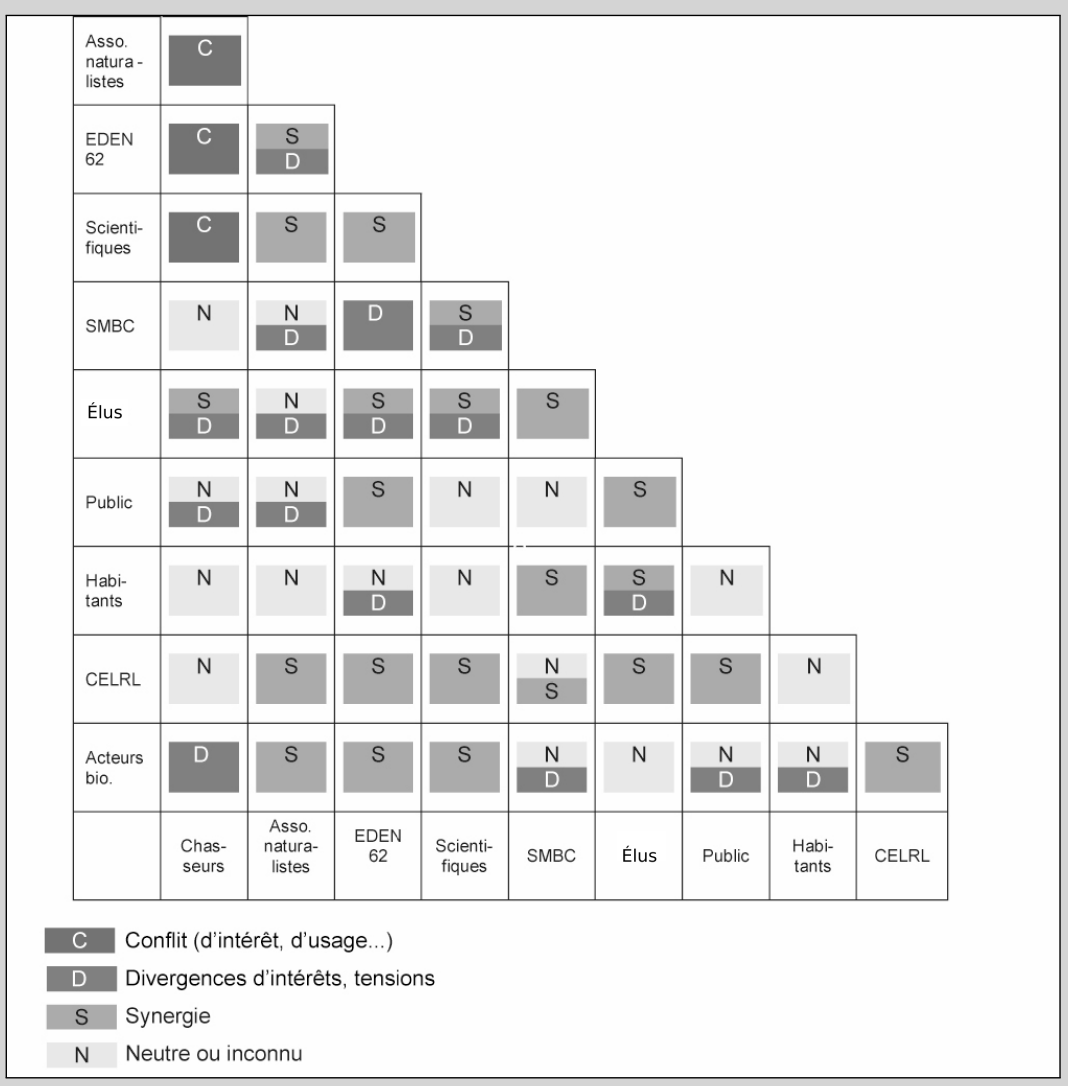

1. Syndicat mixte EDEN 62, gestionnaire de la réserve : financé par la TDENS (taxe départementale des espaces naturels sensibles), mission de préservation et de renforcement de la valeur écologique du site, ainsi que d'accueil du public dans un souci de sensibilisation à l'environnement.

2. Conservatoire de l'espace littoral et des rivages lacustres (CELRL) : propriétaire d'une partie de la réserve, confie la gestion à EDEN 62.

3. Service maritime des ports de Boulogne-sur-Mer et de Calais (SMBC) : gestionnaire du DPM, mission d'aménagement et de conservation du DPM en liaison avec les collectivités concernées. Sur la réserve, ses interventions doivent être soumises au comité consultatif et validées par le préfet.

4. Élus locaux : contribuent au financement et au fonctionnement du site, en principe en accord avec les objectifs de la réserve. Mais, en tant que représentants de leurs administrés, il sont aussi les porte-parole des intérêts locaux (lutte contre l'érosion, développement touristique ou chasse).

5. Habitants du lotissement : bénéficient du cadre de vie offert par la réserve. Préoccupation davantage orientée vers la lutte contre l'érosion de la dune que vers les objectifs de gestion naturaliste du site.

6. «Usagers » : visiteurs de la réserve ou promeneurs traversant la réserve vers la plage. Deux sources de tension : une présence trop massive peut interférer avec la gestion (piétinement intensif et dérangement de l'avifaune); ils sont de fait exclus du secteur de l'estuaire par la pratique de la chasse.

7. Associations de protection de la nature : contribuent à la connaissance du site et à la diffusion de ce savoir, très attachées aux objectifs de la réserve, grande force de mobilisation et de recours juridique, parfois en conflit ouvert avec les chasseurs, les élus ou même le gestionnaire.

8. Chasseurs : effectuent des prélèvements sur la faune et réalisent, au niveau des huttes et des mares artificielles, des modifications volontaires du milieu sans intégration au plan de gestion; entendent pérenniser un droit d'usage et estiment contribuer à la connaissance de la faune sauvage et à sa régulation. Profondes divergences de points de vue avec les gestionnaires et les naturalistes.

9. Les scientifiques : bénévoles ou mandatés par le préfet ou des bureaux d'études, représentent une source de connaissances et une force de proposition qui peuvent contrarier les projets d'autres intervenants (valeur écologique de la réserve) ou susciter la réaction des élus (propositions de choix fondamentaux à long terme pour le lotissement : rechargement en sable ou expropriation).

10. «Acteurs biologiques » (faune et flore) : interagissent avec le milieu physique et avec plusieurs des autres intervenants. Par exemple, l'installation d'une nouvelle espèce modifie le milieu, interpelle les scientifiques et les naturalistes et module les actions du gestionnaire.

nature (plaintes auprès du procureur de la République, manifestations). Après de multiples rebondissements, la condamnation des chasseurs par le tribunal de SaintOmer (juillet 2002), confirmée par la cour d'appel de Douai (juin 2003), met fin à l'utilisation illégale de ces sept huttes. Le problème n'est cependant que partiellement résolu, puisque la chasse reste pratiquée dans les douze huttes situées dans la zone estuarienne, à «l'extérieur $\mathrm{du}$ trait de crayon ». Finalement, en juin 2003, suite au recours de l'association Nord Nature, le tribunal administratif de Lille rétablit les limites officielles de la réserve et précise que le fameux trait de crayon «ne résultait que d'un compromis non valide ». Malgré les procédures de contestation menées par les chasseurs, ce jugement est confirmé en appel (octobre 2003) et en cassation (octobre 2004). Pourtant, au cours de l'été 2004 et sans l'accord du comité consultatif de la réserve, des travaux d'entretien des mares de chasse sont encore effectués à l'aide d'engins lourds (Fig. 2), mettant en évidence, de la part des chasseurs, un sentiment marqué 


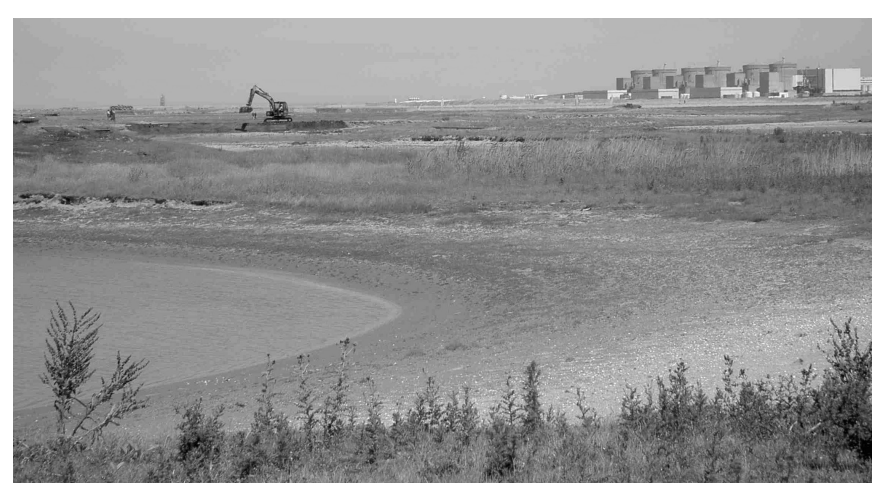

Fig. 2. Travaux d'entretien d'une mare par engins lourds, 2004. (Photo : Michel Delsaut.)

de «droit acquis ». D'ailleurs, ce droit semble être confirmé par les services de l'État, car, pour que chacun puisse "sortir du conflit la tête haute », le sous-préfet accepte de conditionner la démolition des douze huttes à leur reconstruction dans un secteur côtier situé plus à l'ouest (mais soumis à la loi Littoral et présentant une forte valeur biologique).

Cette tolérance de la poursuite d'une activité cynégétique dans une réserve naturelle, outre le fait d'avoir engendré un conflit spectaculaire, a eu plusieurs conséquences concrètes. L'effet direct le plus évident sur la biodiversité réside dans les prélèvements effectués sur l'avifaune sauvage, autant d'oiseaux qui n'ont pu se reproduire ou participer aux chaînes alimentaires. Par ailleurs, l'entretien des mares par des engins de terrassement lourds a provoqué des modifications importantes du substrat et du paysage.

Pour le public, l'interdiction d'accès, du fait de la chasse, à une partie de l'estuaire contribue à créer une image négative et incohérente de la réserve, et éventuellement des réserves naturelles en général. En outre, la présence des chasseurs a relégué la partie estuarienne dans une sorte de zone de non-droit, où même les gardes ne pénétraient pas, ce qui y a interdit tout suivi biologique et toute utilisation didactique pendant 20 ans. Cette situation n'a pas contribué à valoriser la réserve aux yeux des décideurs.

De plus, cette très longue période de tolérance a eu un autre effet, indirect, en donnant aux chasseurs l'illusion $\mathrm{d}^{\prime}$ un droit d'usage ${ }^{2}$ acquis, droit admis par plusieurs autres acteurs. Par exemple, l'habitude a été prise de raisonner sur « le trait de crayon » en tant que limite légale, ce qui amène à la situation paradoxale qu'aujourd'hui ce sont les chasseurs qui se considèrent lésés. Ceci explique peut-être que leurs exigences de « relogement » aient été retenues, alors que le bail de chasse imposait au contraire une remise en état des lieux sans aucune contrepartie.

\footnotetext{
${ }^{2}$ Les droits d'usage sont des droits subjectifs, socialement reconnus et qui ne se rapportent pas à l'appropriation privée du bien (Kirat, 1999).
}

Tout ceci explique également le découragement des autres acteurs, comme certains associatifs ou scientifiques, qui constatent le peu d'effet de leurs avis et propositions.

Par ailleurs, l'impact budgétaire de ces conflits sur les finances publiques n'est pas négligeable (travaux de destruction et reconstruction des huttes, remise en état du site, nombreuses réunions de «conciliation » impliquant plusieurs dizaines de personnes dont plusieurs travaillent pour le service public).

Finalement, l'enlisement du conflit et la tolérance face à une pratique illégale ont occulté, pendant 20 ans, les enjeux de la gestion naturaliste d'une partie essentielle de la réserve.

\section{Conclusion}

Le cas de la Réserve naturelle nationale du Platier d'Oye soulève de nombreuses questions qui révèlent la complexité d'un système de gestion soumis à de multiples contraintes. Plus particulièrement, avec l'exemple du conflit lié à la chasse, on peut s'interroger sur les limites d'une certaine gouvernance (Gaudin, 2002) fondée, d'une part, sur des intérêts locaux fortement soumis aux pressions de lobbies et, d'autre part, sur la recherche de la concertation et du consensus à tout prix (Fortier, 2008). Finalement, sur ce territoire fortement investi, un système de gouvernance fondé sur le principe «gagnant-gagnant » a donné temporairement l'illusion qu'il est possible de résoudre les conflits sans assumer de choix véritable. En réalité, le problème de la chasse révèle de véritables problèmes d'échelles :

- Échelles de valeur : la valeur écologique de la réserve est rarement exprimée en termes économiques (Costanza et al., 1997). Et les différents acteurs n'utilisent pas une "monnaie commune» : par exemple, le gibier pour les chasseurs et les écosystèmes pour les scientifiques ou le gestionnaire.

- Échelles de temps : la gestion de la nature et la gestion intégrée des zones côtières (GIZC) impliquent une échelle de temps longue (de l'ordre du siècle au moins), évidemment difficile à intégrer au niveau local, face à des problématiques de terrain concrètes qui demandent des solutions rapides, à défaut d'être toujours durables.

- Échelles spatiales : il apparaît de plus en plus évident que la préservation de la biodiversité dépasse de loin l'échelle des problématiques locales. La "réussite» d'une réserve naturelle se mesure non seulement localement, mais aussi dans une réflexion et une action globales : intégration à un réseau, échanges avec les autres zones protégées, corridors écologiques (Birard, 2006 ; Moulinas, 2006). 
Or, dans la Réserve naturelle nationale du Platier d'Oye, la notion de préservation de la biodiversité en tant que patrimoine, constitutif de l'intérêt général, est encore mal intégrée et ne trouve pas de place face aux intérêts locaux. «L'intérêt général - ainsi que l'écrit J.-P. Gaudin (2002) - n'a pas disparu, mais il est dilué, revendiqué par tous, démultiplié dans tous les choix locaux. On a inventé l'intérêt général local. Mais quand il est local, comment l'intérêt est-il encore général ? » Alors, le risque existe de voir l'avènement « de pratiques qui emprunteraient surtout aux règles du marché (voire du marchandage), ou qui invoqueraient une démocratie de proximité pour masquer la simple valorisation des rapports de force existants ». En fait, et le cas est manifeste au Platier d'Oye, l'absence de définition des priorités et de débat préalable sur l'intérêt général entraîne la « dilution » de cette notion et sa relégation. Le dialogue local, qui apparaît évidemment comme un impératif, n'est pas simple et semble devoir être appris.

Le but d'une réserve naturelle est de préserver la diversité biologique et de la valoriser en tant que patrimoine commun. Pour que cet objectif, clairement affirmé par les textes juridiques, soit pris en compte, il est nécessaire que les différents acteurs le reconnaissent comme fondamental. Or, la préservation d'espaces de nature et de leur dynamique, si elle est maintenant passée dans le discours, n'a probablement pas encore été intégrée à un niveau suffisant pour que, dans les faits, elle puisse être opposée efficacement aux intérêts particuliers. Car cette préservation peut impliquer l'interdiction de certaines activités ou l'acceptation de la mobilité du trait de côte, ce qui n'apparaît pas encore électoralement gratifiant, ni financièrement rentable, en raison d'une insuffisante prise en compte de la «valeur nature » et d'une réflexion à trop court terme. Contribuer à la diffusion de cette vision auprès des différents acteurs constitue un défi majeur pour l'avenir.

\section{Remerciements}

Les auteurs remercient Alexandre Driencourt (gestionnaire, EDEN 62) pour ses remarques constructives.

\section{Références}

Birard, C., 2006. Corridors : lieux d'échanges, les chemins de la vie, Espaces naturels, 14, 10-11.

Costanza, R., d'Arge, R., de Groot, R., Farber, S., Grasso, M., Hannon, B., Naeem, S., Limburg, K., Paruelo, J., O'Neill, R.V., Raskin, R., Sutton, P., Van den Belt, M., 1997. The value of the world's ecosystem services and natural capital, Nature, $387,253-260$.

Fortier, A., 2008. Quand la concertation produit de l'évitement. La mise en œuvre de Natura 2000 en région Nord-Pas-de-Calais, Développement durable et territoires, http: //developpementdurable.revues.org/document4602.html

Gaudin, J.-P., 2002. Pourquoi la gouvernance?, Paris, Presses de Sciences Po.

Goeldner-Gianella, L., 2007. Perceptions and attitudes towards de-polderisation in Europe: a comparison of five opinion surveys, Journal of Coastal Research, 23, 5, 1218-1230.

IPCC, 2007. Summary for policymakers, Climate Change 2007: The Physical Science Basis. Contribution of Working Group I to the Fourth Assessment Report of the Intergovernemental Panel of Climate Change, http://www.ipcc.ch/pdf/assessment-report/ ar4/wg1/ar4-wg1-spm.pdf

Jeanneaux, P., 2006. Économie de la décision publique et conflits d'usages pour un cadre de vie dans les espaces ruraux périurbains, Développement durable et territoires, http: //developpementdurable.revues.org./document2586.html

Kirat, T. 1999. Économie du droit, Paris, La Découverte.

Meur-Férec, C., Deboudt, P., Morel, V. 2008. Coastal risks in France: an integrated method for evaluating vulnerability, Journal of Coastal Research, 24, sp2, 178-189.

Moulinas, G., 2006. Corridors écologiques. Un nouvel état d'esprit, Espaces naturels, 14, 9.

Reynaud, J.-D., 1999. Le Conflit, la négociation et la règle, Toulouse, Octarès.

Theys, J., 2002. La gouvernance entre innovation et impuissance : le cas de l'environnement, Développement $d u$ rable et territoires, http://developpementdurable.revues.org/ document1523.html 